\title{
Ni ingenuos ni malvados. Enmarcado y estrategias dominantes en la toma de decisiones en contextos de ignorancia
}

Juan Antonio González de Requena Farré*

Doctor en Filosofía, Universidad Complutense de Madrid. Profesor, Escuela de Psicología, Universidad Austral de Chile, Puerto Montt, Chile.

Correo electrónico:

jgonzalez@spm.uach.cl

Recibido: 18 de agosto del 2015

Aprobado: 29 de octubre del 2015

Cómo citar este artículo: González, J.A. (2015). Ni ingenuos ni malvados. Enmarcado y estrategias dominantes en la toma de decisiones en contextos de ignorancia. Pensando Psicología, 11(18), 59-71. doi: http://dx.doi.org/10.16925/pe.v11i18.1219

\section{Resumen}

Introducción: numerosas investigaciones psicológicas sobre decisión asimilan el riesgo y la ignorancia bajo la categoría de decisión en contextos de incertidumbre. Objetivo: en esta investigación se pretenden describir, específicamente, los modos de empleo de algunas reglas de decisión en contextos de ignorancia ante diferentes consecuencias, afectados y bienes. De esta manera, es posible determinar si se dan algunos de los modos de enmarcado de la decisión que se han observado en contextos de riesgo, tales como los considerados por la teoría prospectiva de Kahneman y Tversky. Metodología: se realizó un estudio comparativo de los resultados de un cuestionario con 24 situaciones de apuesta con diferentes bienes, afectados y consecuencias, el cual fue aplicado a una muestra de 232 personas con su consentimiento informado. Resultados: los resultados evidencian diferencias significativas en el empleo de criterios de decisión en contextos de ignorancia, con un claro predominio de la estrategia conservadora. Conclusiones: cabe concluir que, en contextos de decisión bajo ignorancia, se agudiza la aversión a la pérdida y se modifica levemente el efecto de enmarcado observado en decisiones bajo riesgo, pues no necesariamente se arriesga más ante la perspectiva de pérdida.

Palabras clave: contextos de ignorancia, elección racional, enmarcado, reglas de decisión. 


\title{
Neither Naïve nor Evil. Framing and Dominant Strategies in Decision-making in Contexts of Ignorance
}

\begin{abstract}
Introduction: Numerous psychological research studies about decision-making address both risk and ignorance under the category of decision in contexts of uncertainty. Objective: In this research work, the aim is to specifically describe the ways certain decision rules are used in a context of ignorance in the context of different consequences, affected parties and properties. In this way, it is possible to determine whether or not some of the ways of framing a decision that have been observed in contexts of risk actually occur, such as those considered by the prospective theory of Kahneman and Tversky. Methodology: A comparative study of the results was made of the results of a questionnaire with 24 gambling situations with different properties, affected parties and consequences, which was then applied to a sample of 232 people with their informed consent. Results: The results showed significant differences in the use of decision criteria in contexts of ignorance, with a clear predominance of the conservative strategy. Conclusions: It may be concluded that in contexts of decision-making in ignorance, aversion to loss becomes acute and the framing effect observed in low-risk decisions is slightly modified, because more risk is not necessarily run in a perspective of loss.
\end{abstract}

Keywords: contexts of ignorance, rational choice, framing, decision rules.

\section{Nem ingênuos nem malvados. Efeito framing e estratégias dominantes na tomada de decisões em contextos de ignorância}

\section{Resumo}

Introdução: numerosas pesquisas psicológicas sobre decisão assimilam o risco e a ignorância sob a categoria de decisão em contextos de incerteza. Objetivo: nesta pesquisa, pretende-se descrever, especificamente, os modos de emprego de algumas regras de decisão em contextos de ignorância ante diferentes consequências, afetados e bens. Dessa maneira, é possível determinar se são dados alguns modos de efeito framing da decisão que têm sido observados em contextos de risco, tais como os considerados pela teoria prospectiva de Kahneman e Tversky. Metodologia: realizou-se um estudo comparado dos resultados de um questionário com 24 situações de aposta com diferentes bens, afetados e consequências, que foi aplicado a uma amostra de 232 pessoas com seu consentimento informado. Resultados: os resultados evidenciam diferenças significativas no emprego de critérios de decisão em contextos de ignorância, com um claro predomínio da estratégia conservadora. Conclusões: cabe concluir que, em contextos de decisão sob ignorância, se agrava a aversão à perda e se modifica, levemente, o efeito framing observado em decisões de baixo risco, pois não necessariamente se arrisca mais diante da perspectiva de perda.

Palavras-chave: contextos de ignorância, escolha racional, efeito framing, regras de decisão. 


\section{Introducción}

Actualmente, es tal la hegemonía de los enfoques consecuencialistas de la decisión, que se suele identificar la teoría de la decisión con el análisis de las elecciones que las personas realizan a fin de obtener el mejor resultado entre las opciones posibles, dada su preferencia individual (Aguiar, 2004). Bajo esta perspectiva, también designada como teoría de la elección racional, se considera que la racionalidad de la decisión radica en la evaluación de las utilidades esperadas de las distintas opciones, dadas las probabilidades y valores que se le asignan a los distintos resultados. La decisión racional coincidiría con la adopción de aquella opción que permite maximizar la utilidad esperada, esto es, el valor resultante de la ponderación de la preferencia subjetiva y la probabilidad objetiva (Bermúdez, 2009, p. 2). Semejante enfoque de la decisión presupone cierto individualismo metodológico (al partir de la elección individual y del interés propio), así como una concepción netamente instrumental de la acción humana (como cálculo racional de utilidades) (Vidal de la Rosa, 2008).

En el marco de la teoría de la elección racional, toda decisión respondería al siguiente esquema básico: se da una elección entre opciones o actos; existen ciertos deseos, preferencias o utilidades asociados a los resultados de las acciones; los resultados dependen de ciertos estados de cosas y de las creencias del sujeto sobre los escenarios objetivos (Resnik, 1998, p. 25; Hastie, 2001). Si el individuo no dispone de toda la información sobre el estado de cosas en que ha de elegir, nos enfrentamos a decisiones en contexto de ignorancia o incertidumbre (cuando se desconocen las probabilidades de cada resultado), o bien a decisiones en contexto de riesgo (si se pueden asignar probabilidades a los resultados de cada opción) (Aguiar, 2004; Resnik, 1998, p. 37). En el caso de decisiones en contextos de ignorancia, surge cierta anomalía para la teoría de la elección racional, en la medida en que no es posible garantizar que la decisión adoptada sea el único medio de lograr un resultado, ni que sea el medio óptimo de la conducta maximizadora (Elster, 1988, pp. 24-25). Al fin y al cabo, cuando falta información sobre las probabilidades de los posibles estados de cosas, existen diferentes estrategias de decisión potencialmente racionales, entre las cuales cabe destacar las siguientes: el criterio extremadamente optimista, lo cual lleva a escoger la mejor entre las mejores consecuencias (regla maximax); el criterio prudentemente optimista, que procura evitar el arrepentimiento por las oportunidades perdidas y escoge la opción con el mínimo arrepentimiento máximo (regla minimax); o el criterio pesimista y conservador, el cual conduce a elegir el mejor entre los peores resultados posibles (regla maximín) (Resnik, 1998, pp. 56-69).

Desde la formulación inicial de la teoría de la utilidad esperada, por parte de Von Neumann y Morgenstern (1944), Savage (1954) o Luce y Raiffa (1957), han ido multiplicándose las anomalías, paradojas y resultados experimentales que impugnan axiomas de la teoría de la elección racional, y ofrecen modelos más descriptivos del modo en que las personas toman decisiones en situaciones reales (Goldstein y Hogarth, 1997). Así, desde finales de los cincuenta, numerosos cuestionamientos teóricos han afectado las premisas de la teoría de la utilidad esperada. Por ejemplo, Herbert Simon (1955) consideró que la satisfacción de las necesidades personales, es decir, la aceptabilidad de los resultados obtenidos (en vista de nuestros limitados recursos cognitivos) resultaría más importante que la optimización (Plous, 1993, p. 95; Goldstein y Hogarth, 1997). En ese sentido, se ha podido observar cómo la cantidad desmedida de opciones y la elección maximizadora, o las altas expectativas sobre los resultados y la comparación social, afectan el bienestar logrado a través de la toma de decisiones, al aumentar el costo de la búsqueda de información y las posibilidades de experimentar arrepentimiento. Y, a partir de esa premisa, algunos investigadores han distinguido entre personas maximizadoras y satisfactoras en la toma de decisiones, según prefieran la mejor opción o una opción suficientemente aceptable (Schwartz et al., 2002).

Así como se ha reconocido el papel de la evaluación del resultado en la toma de decisiones, Kahneman y Tversky (1983) argumentaron que el modo en que se enmarca la decisión en contexto de riesgo (su enfoque como ganancia o pérdida respecto a cierto punto de referencia), influye en el valor que se le asigna a los distintos resultados, de manera que se arriesgaría menos cuando hay perspectivas de ganancia, o se adoptarían decisiones más arriesgadas cuando las opciones se presentan como pérdidas (Plous, 1993, p. 96). En este sentido, la teoría prospectiva de Kahneman y Tversky (1983; Tversky y Kahneman, 1981) predice cambios en la preferencia bajo contextos de riesgo, dependiendo del marco y perspectiva con que se presentan los problemas de decisión. Por ejemplo, la investigación sobre el efecto de enmarcado ha podido establecer una mayor aversión al riesgo 
cuando la perspectiva es positiva, o sea cuando la situación de decisión pone en juego ganancias, y una mayor propensión al riesgo cuando la perspectiva es negativa o de pérdidas. En otras palabras, la ganancia segura resulta más atractiva que la ganancia probable. Sin embargo, las elecciones se invierten ante la perspectiva negativa, y cabe reconocer cierta aversión a la pérdida: se opta por la pérdida probable en vez de la pérdida segura (Kahneman y Tversky, 1983). A partir del trabajo de Kahneman y Tversky, se ha enriquecido la discusión sobre el modo en que el enmarcado de las opciones afecta la elección racional, así como se ha podido establecer que, además de la perspectiva de ganancia o pérdida, también influyen en la toma de decisiones en contextos de riesgo la probabilidad de los resultados o la magnitud de las recompensas (Kühberger, 1997, p. 131).

Por otra parte, se ha podido establecer que existe cierta asimetría entre las decisiones referentes a uno mismo y aquellas que afectan a otros, de manera que la aversión a la pérdida disminuiría cuando se trata de decidir sobre terceros (Polman, 2012). Esta asimetría en la decisión por uno mismo o por terceros se puede relacionar con las diferencias en la distancia psicológica, con las distintas orientaciones motivacionales (promoción o evitación), con la cantidad de información requerida para decidir, o con el sesgo que estima peor una acción dañina que la inactividad perjudicial, e, incluso, se puede vincular a la posición de poder en que se sitúa quien decide por otros (Polman, 2012).

Existe una profusa investigación sobre los aspectos psicológicos que modulan la decisión. A partir de los sesenta y en el contexto del cuestionamiento al modelo estándar de la teoría de la utilidad esperada, se prestó mayor atención a los aspectos psicológicos y los procesos cognitivos que concurren en la toma de decisiones en contextos no ideales (Goldstein y Hogarth, 1997; Hastie, 2001). Más que la formalización lógico-matemática de los modos de maximización de utilidad por parte de un individuo perfectamente informado, la investigación psicológica de la decisión privilegió la descripción de los aspectos concretos involucrados en la decisión humana: las características de la tarea, el decisor como procesador activo de información y la diversidad de sus respuestas decisionales (León, 1987).

La investigación psicológica sobre la toma de decisiones se empezó a centrar más en la descripción de los procesos de ejecución de las tareas de decisión: los modos de adquisición y elaboración de la información, las limitaciones de la atención y la memoria, la motivación y los componentes afectivos y motivacionales, las reglas de producción sensibles al contexto, así como los juicios inferenciales y los heurísticos (Goldstein y Hogarth, 1997; Hastie, 2001). También se ha indagado la manera como influyen los entornos de decisión y las tareas específicas, o las características internas del decisor (sus creencias, valores, metas y experiencias), las diferencias individuales y grupales, de género y etarias, así como los estilos cognitivos y decisionales, o la competencia decisional y la capacidad de cálculo numérica de quien decide (Weber y Johnson, 2009).

Específicamente, el enmarcado de la decisión ha sido vinculado con las características del procesamiento cognitivo borroso y fluido, el cual se focaliza solo en lo esencial y no en los detalles, así como se ha enfatizado la asimetría al ponderar estímulos positivos y negativos (Kühberger, 1997, pp. 133-135). Asimismo, se ha señalado que la toma de decisiones responde a un repertorio de heurísticos, los cuales procuran más un resultado satisfactorio que la mejor solución posible. Particularmente, los heurísticos responden a situaciones en que no hay suficiente información sobre las probabilidades o en las cuales existen múltiples objetivos posibles. En esos casos, hay heurísticos que acortan el proceso de decisión, como, por ejemplo, tomar lo mejor ante la primera pista discriminatoria disponible, o limitarse a enumerar las señales que favorecen una opción, sin considerar su peso decisional (Gigerenzer, 2008).

Se ha reconocido también que la aversión al riesgo se incrementa cuando la apuesta se enmarca como un cuestionamiento al statu quo, representado por la perspectiva de resultados ciertos; de ese modo, la aversión al riesgo se vincularía al sesgo que lleva a preferir lo que se estima como dado y establecido, esto es, los resultados con certeza (Baron y Ritov, 2009; Ert y Erev, 2013).

Otra línea de investigación relevante para ubicar el nicho del presente trabajo proviene de los estudios sobre los estilos de decisión. Desde finales de los años setenta, la investigación acerca de la toma de decisiones se ha enfocado crecientemente en las diferencias individuales y en los estilos decisionales de las personas. Desde que Harren (1979) distinguió tres estilos básicos en la toma de decisiones (el racional, el dependiente de las expectativas ajenas y el intuitivo), se han concebido diversas clasificaciones de los estilos decisionales. En algunos casos, la clasificación de los estilos se basa en los contextos de 
decisión, o sea la complejidad cognitiva y la orientación de las tareas, que la persona prefiere. A partir de esos criterios, cabe distinguir un estilo directivo y gerencial; un estilo conceptual, flexible e intuitivo; un estilo analítico, intelectual y orientado al control; así como un estilo conductual, sociable y relacional (Rowe y Mason, 1987). En otros casos, la clasificación de los estilos decisionales se fundamenta en los procesos cognitivos desplegados en la toma de decisiones, esto es, en la clase de problema (unidimensional o con múltiples focos) que se aborda, y en el uso de información (máximo o satisfactorio) que requiere la persona que decide. Al considerar esas dimensiones, cabe reconocer un estilo decisivo, directo y eficiente; un estilo jerárquico, analítico e intelectualmente complejo; un estilo flexible, adaptativo y receptivo; o un estilo integrador, participativo y creativo (Driver, Brousseau y Hunsaker, 1990). Otras clasificaciones consideran el carácter adaptativo (presente en los estilos con autoconfianza, así como en el estilo vigilante y exhaustivo de decisión), o el carácter inadaptado de los estilos de decisión (característico de los estilos evasivos que posponen la decisión, de los estilos pánicos que sucumben a la ansiedad al decidir, o de los estilos complacientes y dependientes de la guía ajena) (Mann, Harmoni y Power, 1989).

Toda esta indagación sobre los estilos decisionales se ha traducido en la creación de numerosos instrumentos de evaluación, tales como el inventario de estilos de decisión de Rowe y Mason, o como el General Decision-Making Style (GMDS), que evalúa los estilos racional, intuitivo, dependiente, evitativo y espontáneo (Scott y Bruce, 1995).

Ahora bien, parece razonable intentar clasificar los estilos de decisión no solo por los procesos cognitivos movilizados o por los contextos y tareas abordados. Cabe pensar que existen estilos de decisión diferentes en virtud de los criterios o reglas de decisión (maximización de la ganancia, maximización de las oportunidades, minimización de las pérdidas o resignación a los mínimos), los cuales las personas suelen emplear recurrentemente al tomar decisiones bajo ignorancia. De este modo, se podría cerrar cierto círculo en la investigación sobre la decisión, al indagar los estilos decisionales desde la perspectiva formal y consecuencialista más clásica de la teoría de la elección racional (la cual cuenta con la ventaja de explicitar reglas generales y mecanismos impersonales inherentes a la toma de decisiones). Precisamente, la presente investigación no solo pretende describir los modos de enmarcado de la decisión bajo ignorancia, sino también explorar algunos estilos decisionales desde el punto de vista de los criterios utilizados en distintos problemas de decisión en contexto de ignorancia, y a través de un cuestionario que contempla problemas de decisión con diferentes bienes (primarios, secundarios, monetarios o sociales), diversas consecuencias (ganancia o pérdida) y distintos afectados (uno mismo o terceras personas).

En los contextos de decisión bajo ignorancia, cabe esperar que la aversión a la pérdida - que la teoría prospectiva establece para contextos de riesgose exprese como la estrategia de maximización del peor resultado (es decir, el criterio maximín), particularmente cuando esa estrategia no resulta demasiado costosa en términos de pérdidas (Ert y Erev, 2013). Además, en contextos de ignorancia, se puede anticipar que la inexistencia de información sobre los posibles estados de cosas se traduzca en una alta vigencia del efecto de enmarcado, en razón a la inviabilidad de una representación lógicamente integrada de las opciones que permitiera un cálculo de utilidades esperadas y, así, establecer el óptimo. En ese caso, también cabe pensar que en la decisión bajo ignorancia serán relevantes los heurísticos cognitivos y decisionales que atienden a las indicaciones disponibles y al número de pistas favorables, en lugar de sopesar todos los atributos, aunque quizá ello esté sujeto al marco decisional (Gigerenzer, 2008). Por último, en contextos de ignorancia se puede concebir que existan distintos estilos de decisión, según los tipos de estrategia que emplean en los diferentes marcos situacionales. De este modo, estarían en juego distintas maneras de hacerse cargo de la incertidumbre de los resultados: la prudencia conservadora, el cálculo de oportunidades, el optimismo maximalista y la resignación a mínimos, entre otros. Esta investigación pretende dar cuenta de estas expectativas teóricas en torno a la decisión bajo ignorancia.

Las hipótesis que guían la indagación son cuatro:

- Hipótesis 1. Existen diferencias significativas en la frecuencia de empleo de los distintos criterios de decisión (maximax, maximín, minimax o resignación al valor mínimo) en los diversos contextos de ignorancia.

- Hipótesis 2. Existen diferencias significativas en la frecuencia de empleo de los distintos criterios de decisión, según el modo en que se enmarca la elección desde la perspectiva de sus consecuencias (ganancia o pérdida). 
- Hipótesis 3. Existen diferencias significativas en la frecuencia de empleo de los distintos criterios de decisión, según el tipo de afectado por la decisión (si se trata de uno mismo, o se decide por los otros).

- Hipótesis 4. Existen diferencias significativas en la frecuencia de empleo de los distintos criterios de decisión, según el modo en que se enmarca la elección desde el punto de vista de los bienes propuestos (primarios, secundarios, sociales o monetarios).

\section{Método}

Con el propósito de contrastar con la población chilena los resultados obtenidos en la investigación anglosajona (Goldstein y Hogarth, 1997; Hastie, 2001; Weber y Johnson, 2009), así como con la pretensión de extrapolar algunos marcos de la decisión en contextos de riesgo a la decisión en contextos de ignorancia, se realizó un estudio comparativo a partir de los datos obtenidos en un cuestionario de estrategias de decisión elaborado por los autores de este artículo. A diferencia de los estudios experimentales en la teoría de la decisión (consistentes en situaciones de apuesta en contexto de riesgo, en las cuales el sujeto ha de elegir puntualmente entre opciones que suelen asociarse a cierta probabilidad determinada), la metodología de esta investigación contempló un cuestionario que planteaba un repertorio de elecciones en cada ítem (con distintos bienes, consecuencias y afectados), y no especificó la probabilidad de los resultados en cada situación de apuesta, pues se trataba de establecer las estrategias de decisión en contextos de ignorancia. Esta propuesta metodológica, centrada en la elección reiterada por parte de los sujetos en distintas representaciones de situaciones cotidianas, tiene la ventaja de permitir la evaluación de la coherencia y continuidad de los principios de decisión empleados ante distintos bienes, afectados y consecuencias. Además, suministra muchos más datos sobre las estrategias de decisión, de manera que hace posible un análisis estadístico más confiable.

\section{Muestra}

La muestra de la investigación estuvo conformada por 232 personas chilenas: 31 profesionales con enseñanza superior completa; 154 estudiantes de la Sede Puerto Montt de la Universidad Austral de Chile (81 que cursan la carrera de Ingeniería Comercial y
73 de la carrera de Psicología); finalmente, 47 estudiantes de Enseñanza Media de la provincia de Llanquihue, Chile. Se escogió el programa de Ingeniería Comercial bajo la premisa según la cual en ese campo disciplinar se da cierto adiestramiento en el cálculo de utilidades, mientras que el programa de Psicología cubre un ámbito de estudios en el cual primaría la preocupación por otras personas. De las 232 personas, 137 son de género femenino y 95 de género masculino; las edades van de los 14 a los 63 años, con una media de edad de 22,2 años ( $\mathrm{SD}=7,45)$.

\section{Procedimiento}

Se aplicó un cuestionario autoadministrado de estrategias de decisión en contextos de ignorancia conformado por 24 ítems. Los estudiantes contestaron el cuestionario en una hoja de respuesta que iba acompañada de un consentimiento informado. En el caso de los profesionales, contestaron el cuestionario en línea, a través de una aplicación generada en Google Drive. Este procedimiento de aplicación de cuestionario a través de Internet ha sido avalado por investigadores de la decisión como Baron (2001; Baron y Leshner, 2000).

Cada ítem representaba una situación de elección con cuatro opciones posibles, en cada una de las cuales se obtenían distintas utilidades (según ciertos estados) y se aplicaban distintos principios de decisión. Una de las opciones correspondía siempre al principio maximax (la elección de la opción que contenía la mayor utilidad esperada); otra, al principio minimax (la elección de la opción en que menos oportunidades se pierden o en que el arrepentimiento máximo es menor); otra, al principio maximín (la elección de la opción en que la pérdida sería menor); además, se incluyó una opción con los valores mínimos para la apuesta. En la tabla 1 se exponen un par de ejemplos de cómo se estructuraban los ítems del cuestionario.

El repertorio de ítems incorporaba doce situaciones de decisión en las cuales se ganaba algo y doce en que algo se perdía; doce situaciones de decisión en que el afectado por la decisión era uno mismo y doce en las que se decidía por otras personas. Además, en seis ítems se trataba de valores monetarios; en siete, de valores básicos (alimento o hambre, salud o enfermedad); en siete, de valores secundarios o instrumentales (empleo, entretenimiento, tiempo o vacaciones); en cuatro ítems, de valores sociales (sanción, humillación, repudio o respeto). Las situaciones 
Tabla 1

Ejemplos de la estructuración de los ítems del cuestionario aplicado

\begin{tabular}{|c|c|}
\hline $\begin{array}{l}\text { [Perspectiva positiva al decidir sobre uno } \\
\text { mismo con bienes monetarios en juego] }\end{array}$ & $\begin{array}{l}\text { [Perspectiva negativa al decidir sobre otras } \\
\text { personas con bienes básicos en juego] }\end{array}$ \\
\hline $\begin{array}{l}\text { 1. Ponte en el caso que tienes que elegir un empleo. ¿Cuál de } \\
\text { estos empleos a tiempo parcial escogerías si el pago que recibes } \\
\text { depende del horario que te asignarán? }\end{array}$ & $\begin{array}{l}\text { 14. ¿Qué vacuna decidirías aplicar en una comunidad, sabiendo } \\
\text { que algunas personas enfermarán, según la cepa de virus que } \\
\text { circule? }\end{array}$ \\
\hline $\begin{array}{l}\text { a. En cierto empleo, ganarías } \$ 150.000 \text { mensualmente si se trabaja } \\
\text { en la mañana, y ganarías } \$ 550.000 \text { mensualmente si se trabaja } \\
\text { en la tarde. [estrategia implícita: minimax] }\end{array}$ & $\begin{array}{l}\text { a. Con la primera vacuna, se enfermarán } 5 \text { personas si la cepa es } \\
\text { la de otros años, y se enfermarán } 80 \text { si la cepa es nueva. [estra- } \\
\text { tegia implícita: maximax] }\end{array}$ \\
\hline $\begin{array}{l}\text { b. En determinado empleo, ganarías } \$ 100.000 \text { mensualmente si se } \\
\text { trabaja en la mañana, y ganarías } \$ 700.000 \text { mensualmente si se } \\
\text { trabaja en la tarde. [estrategia implícita: maximax] }\end{array}$ & $\begin{array}{l}\text { b. Con la segunda vacuna, se enfermarán } 20 \text { personas si la cepa } \\
\text { es la de otros años, y se enfermarán } 60 \text { si la cepa es nueva. } \\
\text { [estrategia implícita: minimax] }\end{array}$ \\
\hline $\begin{array}{l}\text { c. En cierto empleo, ganarías } \$ 400.000 \text { mensualmente si se trabaja } \\
\text { en la mañana, y ganarías } \$ 200.000 \text { mensualmente si se trabaja } \\
\text { en la tarde. [estrategia implícita: maximín] }\end{array}$ & $\begin{array}{l}\text { c. Con la tercera vacuna, se enfermarán } 50 \text { personas si la cepa es } \\
\text { la de otros años, y se enfermarán } 35 \text { si la cepa es nueva. [estra- } \\
\text { tegia implícita: maximín] }\end{array}$ \\
\hline $\begin{array}{l}\text { d. En determinado empleo, ganarías } \$ 50.000 \text { mensualmente si se } \\
\text { trabaja en la mañana, y ganarías } \$ 75.000 \text { mensualmente si se } \\
\text { trabaja en la tarde. [estrategia implícita: mínimo] }\end{array}$ & $\begin{array}{l}\text { d. Con la cuarta vacuna, se enfermarán } 50 \text { personas si la cepa es } \\
\text { la de otros años, y se enfermarán } 90 \text { si la cepa es nueva. [estra- } \\
\text { tegia implícita: valor mínimo] }\end{array}$ \\
\hline
\end{tabular}

Nota. En corchetes y cursiva figura el marco de cada ítem y la estrategia que se seleccionaba en cada opción. Elaboración propia.

de decisión planteadas por los ítems se ordenaron de modo aleatorio a fin de no generar efectos de facilitación o interferencia. Cabe destacar que el cuestionario exhibió una alta fiabilidad de escala (un alfa de Cronbach de 0,97).

Se tabularon los datos por decisión, y se trabajó con una planilla Excel de 5568 filas, correspondientes al total de las decisiones emitidas por los 232 sujetos en los 24 ítems del cuestionario. Para contrastar las hipótesis, se analizaron los datos con el programa R en su versión 2,14 (aunque el árbol de clasificación se realizó con spss, versión 21). Por tratarse de variables categóricas, se realizaron tablas de contingencia y se aplicó la prueba estadística $\chi^{2}$, la cual permite establecer diferencias significativas entre frecuencias. Finalmente, se trataron de identificar los estilos de decisión de la muestra chilena a través de las reglas de decisión predominantes y de los enfoques de la decisión (centrados en los valores mínimos o en los valores máximos). Todo esto a partir de una segunda planilla Excel ordenada por sujeto, y en cuyas columnas se recogían las frecuencias de cada criterio (maximax, minimax, maximín, mínimo).

\section{Resultados}

En cuanto a las reglas de decisión más frecuentes en la muestra chilena, se aprecia un predominio del criterio maximín (41,9\%), seguido por el criterio minimax $(28,9 \%)$, el maximax $(19,1 \%)$ y, contra todo pronóstico, se presentó la opción por el mínimo valor esperado en cada apuesta $(9,9 \%)\left(\chi^{2}=1255,05, d f=3\right.$, $p<0,0001)$. Al considerar qué regla era más utilizada por cada sujeto, se observó que, en la mayoría de las personas de la muestra $(58,6 \%)$, predominaba el criterio maximín, seguido por el grupo de personas en que primaba el minimax (23,7\%), el maximax $(7,7 \%)$ o la dupla maximín-minimax $(6 \%)\left(\chi^{2}=624,16, d f\right.$ $=8, p<0,0001)$; el resto de las estrategias o combinaciones de estrategias cubrían el restante 3,9\%. Al agrupar los casos de empleo de reglas centradas en los valores mínimos (maximín y la opción por el valor mínimo) y, por otra parte, las reglas centradas en los valores más elevados (maximax y minimax), se pudo apreciar en la muestra un predominio del enfoque decisional que evalúa los menores valores de cada apuesta (49,1\%), seguido por el enfoque centrado en los máximos (39,6\%), y el enfoque neutro de quienes no se decantan por alguno de los enfoques anteriores $(11,2 \%)\left(\chi^{2}=54,24, d f=2, p<0,0001\right)$. En conclusión, se pudo descartar la hipótesis neutra según la cual no habría diferencias significativas en el empleo de las distintas reglas de decisión en contextos de incertidumbre.

Los análisis estadísticos descriptivos de cada regla de decisión permiten establecer sus distintas medias y cuartiles en la muestra, tal como se aprecia en la tabla 2. 
Tabla 2

Estadísticos descriptivos de cada regla de decisión

\begin{tabular}{lcccccccc}
\hline & M & SD & $\mathbf{0 \%}$ & $25 \%$ & $50 \%$ & $75 \%$ & $100 \%$ & n \\
\hline Maximax & 4,59 & 2,74 & 0 & 2 & 4 & 7 & 12 & 232 \\
Maximín & 10,06 & 3,41 & 3 & 8 & 10 & 12 & 19 & 232 \\
Minimax & 6,94 & 3,04 & 1 & 5 & 7 & 9 & 17 & 232 \\
Mínimo & 2,39 & 2,03 & 0 & 1 & 2 & 4 & 10 & 232 \\
\hline
\end{tabular}

Nota. Se incluye la media, la desviación estándar y los cuartiles. Elaboración propia.

Al considerar si los sujetos de la muestra empleaban los criterios de decisión con valores hasta la media de la muestra o por encima de la media, fue posible reconocer los tipos de decisores más frecuentes en la muestra, tal como se aprecia en la tabla 3.

El resto de decisores hallados comprende los siguientes tipos: el calculador prudente, que recurre por sobre la media a las reglas maximín y minimax $(5,6 \%)$; el buscador de oportunidades resignado $(5,1 \%$ de la muestra), quien recurre por encima de la media a las reglas minimax y a la opción por el mínimo; los distintos tipos de diversificadores que presentan diferentes combinaciones de tres reglas empleadas por encima de la media $(6,8 \%)$; o el extraño caso de los renunciantes $(4,7 \%)$ que optan por el mínimo, por encima de la media.

Para la segunda hipótesis, también se descartó la hipótesis neutra según la cual las consecuencias (ganancia o pérdida) de la elección no enmarcan la regla empleada. Se apreció una diferencia significativa entre los criterios de decisión adoptados, según la perspectiva de ganar o perder $\left(\chi^{2}=165,91, d f=3, p<\right.$ $0,0001)$. Si bien no hay diferencias significativas en el empleo de la regla maximín (que predomina tanto en caso de ganancia como de pérdida, con valores sobre el 41\%), de acuerdo con los residuos, se emplea más la regla maximax cuando lo que está en juego son ganancias $(24,7 \%)$ en vez de pérdidas $(13,5 \%)$, así como se elige más la opción con utilidad mínima en caso de ganancias (11\%) que en caso de pérdida (9\%); por el contrario, se recurre más al criterio minimax en caso de pérdida (34,9\%) que de ganancia (23\%).

En cuanto a la tercera hipótesis, se descartó la hipótesis neutra según la cual el afectado (uno mismo o los otros) no se asocia a la adopción del criterio de decisión, ya que se encontró una diferencia significativa entre los criterios adoptados en cada caso $\left(\chi^{2}\right.$ $=21,02, d f=3, p=0,0001)$. Si bien no hay diferencias significativas en el empleo de la regla maximín (que predomina tanto en caso de decidir por uno o por los demás, con valores sobre el $41 \%$ ), según los residuos, en caso de que se decida por otros se emplea más la regla maximax $(21,4 \%)$, que en caso de que uno mismo sea el afectado (16,8\%); por el contrario, se recurre más al criterio minimax cuando uno es afectado $(30,6 \%)$ que cuando se decide por otros $(27,3 \%)$. $\mathrm{Al}$ considerar conjuntamente las consecuencias y los afectados de la decisión, se aprecian diferencias significativas en el empleo de todas las reglas, tal como queda patente en la tabla 4 .

En concreto, se observa mayor prevalencia de la estrategia maximax cuando está en juego la ganancia de otros que cuando se trata de la pérdida ajena o la ganancia personal. Además, se aprecia mayor empleo de la regla conservadora maximín cuando se trata de la ganancia personal o la pérdida ajena, y se tiende a maximizar las oportunidades con la regla minimax cuando se enfrenta la pérdida personal, así como la

Tabla 3

Los siete estilos de decisor más frecuentes, según su empleo de las reglas de decisión hasta la media o por encima de la media de la muestra

\begin{tabular}{|c|c|c|c|c|c|}
\hline Tipo & Maximax & Maximín & Minimax & Mínimo & Frecuencia \\
\hline El conservador calculador de pérdidas & $\leq 4$ & $>10$ & $\leq 7$ & $\leq 2$ & $13,8 \%$ \\
\hline El oscilante & $>4$ & $\leq 10$ & $\leq 7$ & $>2$ & $13,8 \%$ \\
\hline El calculador de oportunidades & $\leq 4$ & $\leq 10$ & $>7$ & $\leq 2$ & $11,6 \%$ \\
\hline El conservador optimista & $>4$ & $>10$ & $\leq 7$ & $\leq 2$ & $11,2 \%$ \\
\hline El optimista calculador de oportunidades & $>4$ & $\leq 10$ & $>7$ & $\leq 2$ & $10,8 \%$ \\
\hline El conservador resignado & $\leq 4$ & $>10$ & $\leq 7$ & $>2$ & $9 \%$ \\
\hline El optimista arriesgado & $>4$ & $\leq 10$ & $\leq 7$ & $\leq 2$ & $7,3 \%$ \\
\hline
\end{tabular}

Nota. $\chi^{2}=66,77, d f=12, p<0,0001$. Elaboración propia. 
Tabla 4

Frecuencia en porcentaje del criterio de decisión adoptado, dependiendo de las consecuencias y el afectado

\begin{tabular}{lcccc}
\hline & $\begin{array}{c}\text { Ganancia } \\
\text { ajena }\end{array}$ & $\begin{array}{c}\text { Ganancia } \\
\text { personal }\end{array}$ & $\begin{array}{c}\text { Pérdida } \\
\text { ajena }\end{array}$ & $\begin{array}{c}\text { Pérdida } \\
\text { personal }\end{array}$ \\
\hline Maximax & $27,1 \%$ & $22,3 \%$ & $15,7 \%$ & $11,4 \%$ \\
Maximín & $37,6 \%$ & $45,2 \%$ & $45,0 \%$ & $40,1 \%$ \\
Minimax & $25,4 \%$ & $20,5 \%$ & $29,2 \%$ & $40,7 \%$ \\
Mínimo & $9,9 \%$ & $12,0 \%$ & 10,1 & $7,8 \%$ \\
\hline
\end{tabular}

Nota. $\chi^{2}=239,44, d f=9, p<0,0001$. Elaboración propia.

opción por el mínimo prima ante la perspectiva de ganancia personal y se reduce cuando está en juego la pérdida personal.

Respecto a la cuarta hipótesis, también se pudo desechar la hipótesis neutra según la cual el bien en juego no se asocia a la regla de decisión. Se encontró una asociación altamente significativa $\left(\chi^{2}=154,18\right.$, $d f=9, p<0,0001)$ entre el tipo de bien por el que se apostaba y el criterio de decisión empleado. De acuerdo con los residuos, se recurre más a la regla maximax cuando están en juego bienes básicos tales como la comida o la salud $(21,2 \%)$ o dinero $(24,9 \%)$, que cuando se trata de bienes instrumentales como el entretenimiento (14\%), y de bienes sociales como el respeto $(15,6 \%)$. Asimismo, se recurre más a la opción por intentar ganar oportunidades en el caso de los bienes instrumentales $(35,7 \%)$ y de los sociales $(30,7 \%)$, que en el caso de los bienes básicos (28,6\%) o monetarios $(20,4 \%)$; la opción por el mínimo es mayor cuando se trata de dinero $(13,2 \%)$ que cuando se apuesta por bienes sociales (10,7\%), bienes secundarios $(10,2 \%)$ o básicos (6,6\%). Al considerar conjuntamente el tipo de bien y la consecuencia implicada (ganancia o pérdida) se obtienen diferencias significativas en el empleo de reglas de decisión, como se observa en la tabla 5 .
El mayor empleo de la regla maximax se da antela perspectiva de ganancia monetaria y el menor ante la ganancia de un bien social; por el contrario, el mayor empleo del criterio maximín se da en el marco de la ganancia de bienes sociales y el menor, ante la ganancia monetaria. Se recurre más a la regla minimax ante la perspectiva de perder un bien secundario, y menos ante la ganancia monetaria. La opción por el mínimo es mayor cuando se trata de pérdida de dinero y menor ante la pérdida de un bien secundario.

Por último, se llevó a cabo un árbol de clasificación en spss, con el fin de explorar de qué manera las variables predictoras (consecuencia, afectado y bien) se asociaban a la variable criterio (la regla de decisión), y se empleó como método de crecimiento la detección automática de interacciones mediante chi-cuadrado (CHAID). Si bien la bondad predictiva del modelo obtenido es nula - pues solo permite clasificar correctamente un $44,8 \%$ (un $83 \%$ para la regla maximín) - , de acuerdo con el árbol generado la perspectiva resultaría más relevante que el tipo de bien y, finalmente, que el afectado, para la selección de la regla decisional. La regla maximín de decisión bajo ignorancia predomina en casi todos los nodos terminales y, por ende, en la mayoría de los marcos de consecuencias, afectados y bienes que figuraban en el cuestionario. Especialmente, ante la perspectiva de pérdida de bienes básicos personales, con un $65,5 \%\left(\chi^{2}=60,061, d f=3, p<0,0001\right)$. Solo existen tres excepciones: en caso de decisiones concernientes a la ganancia personal de bienes monetarios, la estrategia maximax se impone a las restantes con un $45,3 \%$ $\left(\chi^{2}=45,093, d f=3, p<0,0001\right)$; en caso de decisiones que afrontan una pérdida de bienes básicos ajenos predomina la estrategia minimax con un $40,9 \%$ $\left(\chi^{2}=60,061, d f=3, p<0,0001\right)$; en caso de decisiones en las que está en juego la pérdida personal de bienes

Tabla 5

Frecuencia en porcentaje de reglas de decisión según la consecuencia y el bien implicado

\begin{tabular}{lcccccccc}
\hline & $\begin{array}{c}\text { Ganancia de } \\
\text { bien básico }\end{array}$ & $\begin{array}{c}\text { Ganancia } \\
\text { de bien } \\
\text { secundario }\end{array}$ & $\begin{array}{c}\text { Ganancia } \\
\text { monetaria }\end{array}$ & $\begin{array}{c}\text { Ganancia } \\
\text { de bien } \\
\text { social }\end{array}$ & $\begin{array}{c}\text { Pérdida de } \\
\text { bien básico }\end{array}$ & $\begin{array}{c}\text { Pérdida } \\
\text { de bien } \\
\text { secundario }\end{array}$ & $\begin{array}{c}\text { Pérdida } \\
\text { monetaria }\end{array}$ & $\begin{array}{c}\text { Pérdida } \\
\text { social }\end{array}$ \\
\hline Maximax & 26,7 & 16,6 & 36,5 & 13,8 & 13,9 & 10,6 & 13,4 & 16,2 \\
Maximín & 41,4 & 43,4 & 35,3 & 51,3 & 46,6 & 35,8 & 47,6 & 40,2 \\
Minimax & 24,9 & 26,4 & 16,7 & 20,3 & 33,5 & 48,0 & 24,1 & 34,2 \\
Mínimo & 7,0 & 13,6 & 11,5 & 14,7 & 6,0 & 5,6 & 14,9 & 9,3 \\
\hline
\end{tabular}

Nota. $\chi^{2}=446,31, d f=21, p<0,0001$. Elaboración propia. 
derivados, también prima la estrategia minimax con un $56,5 \%\left(\chi^{2}=49,871, d f=3, p<0,0001\right)$.

\section{Discusión}

Cabría pensar que la mayoría de las decisiones cotidianas se producen en contextos de ignorancia (pues no se les pueden atribuir probabilidades a los resultados). Sin embargo, gran parte de la literatura anglosajona sobre decisión se ha centrado en las decisiones en contexto de riesgo y, cuando se hace cargo de la decisión en contexto de ignorancia, se enfoca sobre todo en las diferencias individuales en la reacción a la ambigüedad y la preferencia o rechazo de la incertidumbre. Se ha hallado cierta correlación y un factor disposicional común entre la aversión al riesgo y el rechazo de la incertidumbre (Lauriola, Levin y Hart, 2007). Esta investigación partió del propio marco de la teoría de la elección racional a fin de establecer diferencias significativas en el empleo de algunas de las reglas de decisión en contextos de ignorancia (el maximax, el maximín o el minimax), en lugar de realizar una aproximación psicológica a los procesos y disposiciones implicadas en la decisión.

En este sentido, se logró contrastar cada una de las cuatro hipótesis propuestas, y se encontraron diferencias significativas en el empleo de los distintos criterios de decisión bajo ignorancia. En términos generales, se observó una prevalencia de la decisión basada en la minimización de pérdidas y del enfoque decisional centrado en los valores mínimos de la apuesta. Este predominio de la opción por la minimización de pérdidas y por los valores mínimos parece adecuarse a un tipo de situación de decisión en que se ignoran las probabilidades de los estados y, por tanto, no se pueden calcular las utilidades esperadas ni procurar la optimización. La conducta decisional no es siempre maximizadora, sino que frecuentemente procura solo un resultado satisfactorio (Schwartz et al., 2002), y aparentemente la decisión bajo ignorancia aporta un escenario en que razonablemente se prefiere una satisfacción suficiente, pues no se puede calcular el óptimo.

Además, nuestra investigación evidenció cierta asociación entre el enmarcado de la situación de apuesta y la regla de decisión adoptada. La decisión en contexto de riesgo evidencia la importancia de la perspectiva de ganancia o pérdida que enmarca la decisión, particularmente en el sentido de cierta aversión a la pérdida (Kahneman y Tversky, 1983; Kühberger, 1997). También en la decisión en contexto de incertidumbre hay un enmarcado por las consecuencias (ganancia o pérdida) de la regla de decisión adoptada, de manera que, cuando está en juego una pérdida, se calculan oportunidades, pero se da más la maximización riesgosa o la resignación a los mínimos en caso de perspectiva positiva. Aparentemente, cuando alguien enfrenta una perspectiva de ganancia, no tiende a sopesar tanto los alcances de la decisión, sino que más bien recurre a una estrategia enfocada solo en el mejor resultado disponible. En cambio, quien enfrenta la toma de decisiones en una perspectiva de pérdida, parece detenerse a sopesar con más detenimiento los distintos resultados de la decisión que se adoptará. En el contexto de la teoría prospectiva, las investigaciones en toma de decisiones han estudiado básicamente situaciones de riesgo (que asignan probabilidades definidas a los resultados), y en este sentido hallaron sesgos decisionales tales como la aversión al riesgo ante un enmarcado de ganancia, o la propensión al riesgo ante la perspectiva de pérdida. En situaciones de ignorancia como las abordadas en este estudio (en las que se desconoce la probabilidad de los resultados), las personas tienden generalmente a adoptar una estrategia conservadora.

Ahora bien, del mismo modo que en situaciones de riesgo se escoge el resultado seguro cuando la perspectiva es de ganancia, y se opta por procesar las probabilidades y apostar al riesgo ante la perspectiva de pérdida, en el caso de los contextos de ignorancia se escoge el resultado más disponible, la recompensa más abultada y llamativa (estrategia maximax) cuando la perspectiva es de ganancia (sobre todo, de dinero), y se prefiere sopesar cautelosamente la información sobre las oportunidades en escenario de pérdida personal de bienes secundarios o en el marco de pérdidas ajenas de bienes básicos. Así, en los contextos de decisión bajo ignorancia, se acentúa la aversión a la pérdida y se opta por no arriesgar demasiado, eligiendo el mejor de los peores escenarios. Sin embargo, ante la perspectiva negativa de perder ciertos bienes, resulta posible encontrar la estrategia de minimizar las oportunidades perdidas.

Por otra parte, el efecto de enmarcado por las consecuencias resulta tan significativo como el enmarcado por el tipo e importancia del bien involucrado en la apuesta, de modo que la aversión a la pérdida depende de las valoraciones del resultado, como también ha establecido la indagación en decisión en contextos de riesgo (Ert y Erev, 2013). Así como en decisión en contextos de riesgo se ha observado una asimetría a la hora de decidir por uno mismo o por 
terceros (Polman, 2012), también en contextos de ignorancia se encuentra una diferencia significativa en el empleo de las reglas de decisión, dependiendo del afectado. Aunque generalmente el individuo es conservador, opta por maximizar y arriesgar más cuando decide por otros, y calcula más las oportunidades cuando es él el afectado. Se da así cierta asimetría en la toma de decisiones dependiendo del afectado, lo cual podría cuestionar las exigencias de consistencia y continuidad de la racionalidad al momento de tomar decisiones. El carácter limitado de la racionalidad decisional bajo ignorancia se pone de manifiesto particularmente al considerar hasta qué punto se encuentra asociada la estrategia a las distintas variables que enmarcan la decisión (perspectiva, bien y afectado), sin que pueda anticiparse el comportamiento decisional a través de alguna jerarquización de las variables predictoras, tal como evidenció el árbol de clasificación.

Respecto a los estilos dominantes de decisión en contextos de ignorancia, fue posible describir algunos perfiles de decisor (el conservador calculador de pérdidas, el oscilante, el calculador de oportunidades, el conservador optimista, el optimista calculador de oportunidades, el conservador resignado, el optimista arriesgado, el calculador prudente, entre otros), a partir de la frecuencia de empleo de cada estrategia en relación con la media. También, este panorama de tipos de decisores exhibe una gran dispersión y avala la idea de una racionalidad limitada y sensible al contexto en la decisión bajo ignorancia.

Pese a haber trabajado en el marco conceptual de la teoría de la elección racional, esta investigación cuestiona algunos de sus supuestos formales relativos a la consistencia lógica de las decisiones de un grupo humano. Las diferencias individuales y los cambios de criterio según los contextos específicos de decisión desdibujan la imagen de un decisor perfectamente racional y coherente, el cual mantiene sus reglas de decisión constantes en distintos escenarios de apuesta. En ese sentido, también resulta cuestionado el homo oeconomicus que presupone la teoría económica, esto es, la construcción del individuo económico como un maximizador de utilidades o, al menos, como un calculador racional adecuadamente informado y consistente en sus propósitos (Pearce, 1999). Por último, si admitimos que el inteligente busca beneficio tanto para sí mismo como para los otros, que el malvado procura el beneficio para sí y el daño para terceros, que el ingenuo provoca el beneficio ajeno sin beneficio propio, y que el estúpido provoca el daño ajeno y el daño propio (Cipolla, 2009), de esta investigación podría concluirse que el hombre no es particularmente estúpido, santo o malvado.

En términos generales procura minimizar la pérdida tanto propia como ajena, aunque utiliza de modo flexible los criterios de decisión, dependiendo de los bienes, la consecuencia y el afectado: es más cuidadoso al decidir sobre la pérdida ajena (especialmente, si se trata de bienes básicos), y más optimista al apostar por el beneficio propio o ajeno (particularmente, ante bienes monetarios). De igual forma, procura no perder oportunidades cuando enfrenta la perspectiva de pérdidas personales de bienes secundarios, o bien decide más como satisfactor resignado que opta por el mínimo, que cuando la decisión afecta su ganancia personal.

Las conclusiones de esta investigación -más bien exploratoria- están sujetas a una triple reserva por la limitación de la muestra, ya que se basa solo en complejas elecciones simuladas, como si se tratase de apuestas en un contexto real y, finalmente, por la necesidad de una validación estadística del cuestionario aplicado. La diferencia entre la decisión basada en la experiencia cotidiana y aquella simplemente descrita en términos de posibilidades teóricas, podría afectar la preferencia, al introducir una asimetría informacional que llevaría a pensar que algunos estados de cosas son prácticamente imposibles (Hadar y Fox, 2009). Pese al intento que se ha realizado por precisar un contexto específico de decisión, caracterizando la decisión bajo ignorancia como un tipo de incertidumbre distinta de la que se asocia al riesgo, podría observarse que resulta preciso demarcar mejor en este tipo de investigaciones lo que se entiende por riesgo o incertidumbre, y eventualmente distinguir la propensión o aversión al riesgo y, por otra parte, el efecto de la incertidumbre atribuible a la falta de información. Además, habría que establecer claramente cuáles son los rasgos psicológicos e influencias situacionales que modulan la propensión al riesgo (Fox y Tannenbaum, 2011). Se trata de direcciones que ha de asumir la futura investigación en teoría de la decisión en contextos de incertidumbre, de manera que sea posible estudiar en profundidad la relación que existe entre las estrategias en el empleo de criterio de decisión y los estilos de decisión basados en procesos psicológicos.

\section{Agradecimientos}

Agradezco a mi colega René Gallardo por su incondicional apoyo en esta investigación y por expresarme 
sus sagaces inquietudes. El profesor Pablo Pérez de la Escuela de Psicología de la Universidad Austral de Chile (UACH) me brindó colaboración en la aplicación del cuestionario. También doy las gracias a las estudiantes María Rosa Franz y Karina Astorga por su ayuda en la tabulación de los datos.

\section{Referencias}

Aguiar, F. (2004). Teoría de la decisión e incertidumbre: modelos normativos y descriptivos. Empiria. Revista de Metodología de Ciencias Sociales, 8, 139-160. doi: http://dx.doi.org/10.5944/empiria.8.2004.982

Baron, J. (2001). Confusion of group-interest and self-interest in parochial cooperation on behalf of a group. Journal of Conflict Resolution, 45, 283-296. doi:10.1177/0022002701045003002

Baron, J. y Leshner, S. (2000). How Serious Are Expressions of Protected Values? Journal of Experimental Psychology: Applied, 6(3), 183-194. doi: 10.1037//1076898X.6.3.183

Baron, J. y Ritov, I. (2009). Protected Values and Omission Bias as Deontological Judgments. En D. M. Bartels, C. W. Bauman, L. J. Skitka, y D. L. Medin (Eds.), The Psychology of Learning and Motivation (pp. 133-167). San Diego: Academic Press.

Bermúdez, J. L. (2009). Decision Theory and Rationality. New York: Oxford University Press.

Cipolla, C. (2009). Allegro ma non troppo. Barcelona: Crítica.

Driver, M. J., Brousseau, K. E. y Hunsaker, P. L. (1990). The Dynamic Decision Maker. New York: Harper \& Row.

Elster, J. (1988). Uvas amargas. Sobre la subversión de la racionalidad. Barcelona: Península.

Ert, E. y Erev, I. (2013). On the Descriptive Value of Loss Aversion in Decisions under Risk: Six Clarifications. Judgment and Decision Making, 8(3), 214-235. doi: $10.2139 /$ ssrn. 1012022

Fox, C. R. y Tannenbaum, D. (2011). The elusive search for stable risk preferences. Frontiers in Psychology, 2: 298. Recuperado de http://fox-lab.org/elusive-search-for-stable-risk-preferences/ doi: 10.3389/ fpsyg.2011.00298

Gigerenzer, G. (2008). Why Heuristics Work. Perspectives on Psychological Science, 3(1), 20-29. doi: 10.1111/j.17456916.2008.00058.x

Goldstein, W. M. y Hogarth, R. M. (1997). Judgment and Decision Research: Some Historical Context. En W. M. Goldstein y R. M. Hogarth (Eds.), Research on
Judgment and Decision Making: Currents, Connections, and Controversies (pp. 3-65). Cambridge: Cambridge University Press.

Hadar, L. y Fox, C. R. (2009). Information asymmetry in decision from description versus decision from experience. Judgment and Decision Making, 4(4), 317-325.

Harren, V. A. (1979). A Model of Career Decision Making for College Students. Journal of Vocational Behavior, 14, 119-133. doi: 10.1016/0001-8791(79)90065-4

Hastie, R. (2001). Problems for Judgment and Decision Making. Annual Review of Psychology, 52, 653-683. doi: 10.1146/annurev.psych.52.1.653

Kahneman, D. y Tversky, A. (1983). Choices, Values, and Frames. American Psychologist, 39(4), 341-350. doi: 10.1037/0003066X.39.4.341

Kühberger, A. (1997). Theoretical Conceptions of Framing Effects in Risky Decisions. En R. Ranyard, W.R. Crozier y O. Svenson (Eds.), Decision Making: Cognitive Models and Explanations (pp. 128-144). London: Routledge.

Lauriola, M., Levin, I. P. y Hart, S. S. (2007). Common and Distinct Factors in Decision Making under Ambiguity and Risk: A Psychometric Study of Individual Differences. Organizational Behavior and Human Decision Processes, 104, 130-149. doi: 10.1016/j.obhdp.2007. 04.001

León, O. G. (1987). La toma de decisiones individuales con riesgo desde la psicología. Infancia y aprendizaje, 30, 81-94.

Luce, R. y Raiffa, H. (1957). Games and Decisions. New York: Wiley.

Mann, L., Harmoni, R. y Power, C. (1989). Adolescent Decision-making, the Development of Competence. Journal of Adolescence, 12, 265-278. doi: 10.1016/01401971(89)90077-8

Pearce, D. W. (Ed.) (1999). Diccionario Akal de Economía moderna. Madrid: Akal.

Plous, S. (1993). The Psychology of Judgment and Decision Making. New York: McGraw-Hill.

Polman, E. (2012). Self-Other Decision Making and Loss Aversion. Organizational Behavior and Human Decision Processes, 119, 141-150. doi: 10.1016/j.obhdp.2012.06.005

Resnik, M. D. (1998). Elecciones. Una introducción a la teoría de la decisión. Barcelona: Gedisa.

Rowe, A. J. y Mason, R. O. (1987). Managing with Style: A Guide to Understanding, Assessing, and Improving Decision Making. San Francisco: Jossey-Bass. 
Savage, L. J. (1954). The Foundations of Statistics. Nueva York: Dover Press.

Scott, S. G. y Bruce, R. A. (1995). Decision Making Style: the Development and Assessment of a New Measure. Educational and Psychological Measurement, 55, 818831. doi: $10.1177 / 0013164495055005017$

Schwartz, B., Ward, A., Monterosso, J., Lyubomirsky, S., White, K. y Lehman, D. R. (2002). Maximizing versus Satisficing: Happiness is a Matter of Choice. Journal of Personality and Social Psychology, 83, 1178-1197. doi: 10.1037//0022-3514.83.5.1178

Simon, H. (1955). A Behavioral Model of Rational Choice. Quarterly Journal of Economics, 69, 99-118. doi: $10.2307 / 1884852$
Tversky, A. y Kahneman, D. (1981). The Framing of Decisions and the Psychology of Choice. Science, New Series, 211(4481), 453-458. doi: 10.1126/ science. 7455683

Vidal de la Rosa, G. (2008). La Teoría de la Elección Racional en las ciencias sociales. Sociológica, 23(67), 221-236.

Von Neumann, J. y Morgenstern, O. (1944). Theory of Games and Economic Behavior. Princeton: Princeton University Press.

Weber, E. U. y Johnson, E. J. (2009). Mindful Judgment and Decision Making. Annual Review of Psychology, 60, 53-85. doi: 10.1146/ annurev.psych.60.110707.163633 